\title{
Pré-condicionamento isquêmico e desempenho: há viabilidade/racionalidade na sua aplicação?
}

Ischemic preconditioning and performance: is there feasibility/rationality in its application?

\author{
Moacir Marocolo ${ }^{1}$ \\ Gustavo Ribeiro da Mota ${ }^{2, *}$
}

\section{Resumo}

O objetivo deste trabalho foi analisar criticamente a racionalidade da aplicação do pré-condicionamento isquêmico (IPC) como potencial recurso ergogênico visando melhoria do desempenho humano. $O$ contexto histórico e o estado atual são apresentados e discutidos. A utilização do IPC como recurso ergogênico ainda é incerta e necessita resultados mais consistentes para se estabelecer definitivamente.

Palavras-chave: ergogênico, esportes, oclusão sanguínea.

\section{Abstract}

This study aimed to analyze critically the rationale for the application of ischemic preconditioning (IPC) as a potential ergogenic aid aiming at improving human performance. The historical context and the current state are presented and discussed. The application of the IPC as an ergogenic aid is still unclear and requires more consistent results to establish itself definitively.

Key-words: ergogenic, sports, blood flow, occlusion.

Programa de Pós-Graduação em Educação Física, Universidade Federal do Triângulo Mineiro, Av. Tutunas, 490, Tutunas, CEP 38061 500, Uberaba, MG, Brazil. e-mail: grmotta@gmail.com

Conflito de interesses

Os autores declararam não haver conflito de interesses.

Processo de arbitragem 


\section{Introdução}

A busca pelo aumento do desempenho físico data dos tempos da Grécia antiga, onde até mesmo aos animais eram dados alimentos como mel e ervas, por exemplo, objetivando melhorar o desempenho'. Ao longo de todo esse tempo, diversas estratégias foram empregadas $e$, principalmente durante o século XX, a viabilização do uso de testosterona e de seus derivados sintéticos pode ser considerada como um dos fatores responsáveis pelo aumento do desempenho em modalidades de força e potência. Tal fato ocorreu (e pode ocorrer), mesmo com a divulgação explícita de seus efeitos deletérios ${ }^{2,3}$.

Contudo, devido à proibição do uso de várias substâncias nas competições esportivas, seja pela criação de legislações específicas, aliada aos relatos de efeitos deletérios do uso de anabolizantes $^{3-5}$, diferentes estratégias têm sido adotadas por treinadores e cientistas do esporte com intuito de aperfeiçoar o desempenho físico ${ }^{1,6,7}$. Uma recente estratégia que despertou o interesse da comunidade científica internacional é conhecida como ischemic preconditioning (IPC) ou, em português, précondicionamento isquêmico ${ }^{8}$. O objetivo deste trabalho foi analisar criticamente a racionalidade da aplicação do IPC como recurso ergogênico no contexto de potencial melhoria do desempenho humano.

\section{O que é IPC?}

Basicamente, o IPC consiste na repetição de ciclos (de 1 a 4 ciclos normalmente) de oclusão (pressões variam, mas normalmente $\sim 220 \mathrm{mmHg}$ ) e reperfusão do fluxo sanguíneo muscular, por meio da aplicação de torniquete (s), na parte proximal das coxas ou dos braços. Os protocolos utilizados apresentam períodos de oclusão/reperfusão que variam de 2 a 5 minutos ${ }^{9}$, mas 5 minutos de oclusão/reperfusão é o tempo mais comum encontrado na literatura de IPC relacionado ao desempenho $0^{8,10}$. O tempo ideal entre o fim da manobra (IPC) e o início do teste ainda não é consenso e varia entre 5 e 90 minutos na maioria dos estudos relacionados ao desempenho ${ }^{8}$. Apesar disso, autores sugerem que o efeito ergogênico agudo ocorreria dentro de 45 minutos a partir do fim da manobra ${ }^{8,11,12}$.

\section{História do IPC e suas aplicações ao desempenho}

O IPC foi originalmente aplicado com objetivo de proteção tecidual e, o primeiro estudo publicado, ainda na década de 1980, mostrou redução de aproximadamente $75 \%$ na área de infarto em modelo animal, quando da aplicação prévia do IPC ${ }^{13}$. Desde então, muitos estudos têm mostrado a efetividade do IPC para proteção tecidual tanto miocárdica quanto em diversos outros órgãos e tecidos ${ }^{14}$.

Esse efeito potencial despertou a atenção dos pesquisadores da ciência do esporte e, no final da década de 1990 e início dos anos 2000, estudos mostraram efeitos benéficos do IPC sobre o desempenho ${ }^{9,15,16}$. Ressaltamos que, apesar da "explosão" de publicações científicas sobre IPC e desempenho ocorrer a partir do estudo de Libonati e seus colegas em 2001, na verdade desde 1958 fisiologistas Alemães testaram o IPC como recursos ergogênico potencial e encontraram resultados controversos ${ }^{8}$. Porém, considerando a partir do "de novo" de Libonati e colaboradores, estudos relatam melhoria ou nenhum efeito agudo do IPC sobre o desempenho em atividades como ciclismo ${ }^{17}$, sprints, natação ${ }^{18}$, treinamento resistido $^{19,20}$, futebol ${ }^{21}$ e remo em apneia ${ }^{22}$. Também surgiram trabalhos tentando verificar efeito ergogênico do IPC sobre o processo de recuperação do desempenho ${ }^{7,23,24}$, com achados igualmente inconclusivos.

\section{Métodos de investigação e interpretação dos resultados}

Tomando como referência os estudos que investigaram os efeitos do IPC sobre o desempenho físico podemos sugerir que os efeitos em diferentes modalidades são nulos ou triviais ${ }^{8}$ e podem ser menores que o próprio erro da medida ${ }^{25}$. Nesse contexto, os estudos utilizam tanto a estatística "tradicional", reportando testes comparativos para diferenças entre médias (testes $\mathrm{t}$, análise de variância) e/ ou a análise do tamanho do efeito. Mesmo quando os pesquisadores optam por um ou outro, os resultados são pouco expressivos e permitem interpretação ampla e muitas vezes subjetiva ${ }^{8,19,25}$. Assim, mais estudos precisam ser conduzidos para analisar diferentes situações e obtermos conclusões mais definitivas. Talvez a configuração da aplicação ideal ainda não foi encontrada para o esporte/desempenho. Isto é, número de ciclos oclusão/reperfusão, tempo de oclusão/reperfusão, pressões ótimas para ocluir ou simular (sham/placebo), tempo entre o final do protocolo e início do exercício/teste ${ }^{19}$, bem como ferramentas para detectar eventuais "respondedores e não respondedores"10.

Em relação ao esporte, devemos considerar a complexidade envolvida, por exemplo: resultado benéfico em teste de corrida de 5-km (para corredores dessa distância) é mais relevante do que eventual melhoria em determinado teste físico para futebolistas. Simples testes físicos, mesmo que amplamente recomendados, validados e utilizados em diferentes contextos do futebol como os diferentes subtipos de "YoYo" 26,27 , apesar do valor real que eles apresentam, não podem minimizar a complexidade envolvida no "desempenho" desta modalidade ${ }^{28}$. Fatores inerentes ao contexto do jogo como, leitura de jogo/tomada de decisão $0^{29}$, variações na posse de bola $^{30}$, por exemplo, podem influenciar a dinâmica de atividades físicas envolvidas. Portanto, análises mais holísticas ${ }^{28,30}$ e específicas ao subtipo de modalidade ${ }^{31-35}$ são desejáveis.

Outro ponto ressaltado em alguns estudos é que o efeito do IPC poderia ter base motivacional ${ }^{18,20}$, visto que alguns deles mostram que a aplicação da manobra de IPC repetidas vezes minimiza o desempenho físico ${ }^{19,36}$ ao longo do tempo. O efeito placebo, altamente reconhecido em estudos sobre o desempenho humano ${ }^{37}$, portanto, precisa ser controlado e considerado na interpretação e tomada de decisão sobre qualquer estratégia ${ }^{19,25}$

O tempo entre a manobra de IPC e o início do exercício ou teste também deve ser analisado, visto que estudos oriundos da fisiologia clínica propõem que os benefícios do IPC podem durar mais que 24 horas $^{14}$. Assim, testes para determinar se o IPC poderia colaborar com o resultado posterior (por exemplo: 6 horas depois da aplicação) são direções futuras de pesquisa sobre este tópico promissor, mas ainda inconclusivo. Considerando que recente revisão sistemática sobre IPC e desempenho sugere que seus efeitos mais proeminentes ocorrem em atividades glicolíticas e aeróbias ${ }^{10}$, futuros trabalhos poderiam avaliar melhor os efeitos do IPC em diferentes modalidades, como, por exemplo, esportes de combate ${ }^{38-40}$ e exercícios resistidos ${ }^{4-44}$ (importante contribuição glicolítica) e diferentes modalidades de dança ${ }^{45}$ que possuem significativo grau de contribuição aeróbia, dentre outras possibilidades.

\section{Considerações finais}

Os estudos utilizando IPC e desempenho, em geral, mostram resultados pouco expressivos e devem ser interpretados, portanto, com prudência. $O$ fato de que as melhorias em decorrência da aplicação do IPC podem ser menores que o erro de medições em alguns teste ${ }^{25}$ é passível de questionamento. Tanto a estatística tradicional quanto análises de inferência baseada na magnitude (exemplo: tamanho do efeito), podem ser utilizadas para a comparação dos resultados. Porém, cautela é recomendada considerando principalmente o significado fisiológico desses resultados, o contexto do teste físico utilizado, o nível de treinamento dos participantes do estudo em questão (por exemplo: atletas de elite ou amadores), o tipo de exercício e, ainda, a complexidade relacionada à especificidade da modalidade esportiva em questão.

\section{Financiamento}

Gerais.

Fundação de Amparo a Pesquisa do Estado de Minas

\section{Referências}

1. Marocolo M. Ischemic preconditioning as a measure to enhance performance in sports. Deutschland: Physiologie una Anatomie, Deutschen Sporthochschule Köln; 2016.

2. Barbosa Neto O, Abate DT, Marocolo Junior M, et al. Exercise training improves cardiovascular autonomic activity and attenuates renal damage in spontaneously hypertensive rats. Journal of sports science \& medicine. 2013;12(1):52-59. 
3. Medei E, Marocolo M, Rodrigues Dde C, et al. Chronic treatment with anabolic steroids induces ventricular repolarization disturbances: cellular, ionic and molecular mechanism. Journal of molecular and cellular cardiology. Aug 2010;49(2):165-175.

4. Barbosa Neto O, da Mota GR, De Sordi CC, et al. Long-term anabolic steroids in male bodybuilders induce cardiovascular structural and autonomic abnormalities. Clinical autonomic research : official journal of the Clinical Autonomic Research Society. Oct 102017.

5. Neto OB, de Sordi CC, da Mota GR, Marocolo M, Chriguer RS, da Silva VJD. Exercise training improves hypertension-induced autonomic dysfunction without influencing properties of peripheral cardiac vagus nerve. Autonomic neuroscience : basic \& clinical. Dec 2017;208:66-72.

6. Russell M, West DJ, Harper LD, Cook CJ, Kilduff LP. Half-time strategies to enhance second-half performance in team-sports players: a review and recommendations. Sports Med. Mar 2015;45(3):353-364.

7. Garcia CA, da Mota GR, Marocolo M. Cold Water Immersion is Acutely Detrimental but Increases Performance Post-12 h in Rugby Players. International journal of sports medicine. Jul 2016;37(8):619-624.

8. Marocolo M, da Mota GR, Simim MA, Appell Coriolano HJ. Myths and Facts About the Effects of Ischemic Preconditioning on Performance. International journal of sports medicine. Feb 2016;37(2):87-96.

9. Libonati JR, Howell AK, Incanno NM, Pettee KK, Glassberg HL. Brief muscle hypoperfusion/hyperemia: an ergogenic aid? Journal of strength and conditioning research. Aug 2001;15(3):362-366.

10. Incognito AV, Burr JF, Millar PJ. The Effects of Ischemic Preconditioning on Human Exercise Performance. Sports Med. Apr 2016;46(4):531-544.

11. Bailey TG, Jones H, Gregson W, Atkinson G, Cable NT, Thijssen DH. Effect of ischemic preconditioning on lactate accumulation and running performance. Medicine and science in sports and exercise. Nov 2012;44(11):2084-2089.

12. Patterson SD, Bezodis NE, Glaister M, Pattison JR. The Effect of Ischemic Preconditioning on Repeated Sprint Cycling Performance. Medicine and science in sports and exercise. Aug 2015:47(8):1652-1658.

13. Murry CE, Jennings RB, Reimer KA. Preconditioning with ischemia: a delay of lethal cell injury in ischemic myocardium. Circulation. Nov 1986;74(5):1124-1136.

14. Ikonomidis JS, Tumiati LC, Weisel RD, Mickle DA, Li RK. Preconditioning human ventricular cardiomyocytes with brief periods of simulated ischaemia. Cardiovascular research. Aug 1994;28(8):1285-1291.

15. Howell AK, Gaughan JP, Cairns MA, Faigenbaum AD, Libonati JR. The effect of muscle hypoperfusion-hyperemia on repetitive vertical jump performance. Journal of strength and conditioning research. Nov 2001:15(4):446-449.

16. Libonati JR, Cox M, Incanno $\mathrm{N}$, et al. Brief periods of occlusion and reperfusion increase skeletal muscle force output in humans. Cardiologia. Dec 1998;43(12):1355-1360.

17. Paixao RC, da Mota GR, Marocolo M. Acute Effect of Ischemic Preconditioning is Detrimental to Anaerobic Performance in Cyclists. International journal of sports medicine. Jul 102014

18. Marocolo M, da Mota GR, Pelegrini V. Appell Coriolano HJ. Are the Beneficial Effects of Ischemic Preconditioning on Performance Partly a Placebo Effect? International journal of sports medicine. Oct 2015;36(10):822-825.

19. da Mota GR, Marocolo M. The Effects of Ischemic Preconditioning on Human Exercise Performance: A Counterpoint. Sports Med. Oct 2016;46(10):1575-1576.

20. Marocolo M, Willardson JM, Marocolo IC, da Mota GR, Simao R, Maior AS. Ischemic Preconditioning and Placebo Intervention Improves Resistance Exercise Performance. Journal of strength and conditioning research. May 2016;30(5):1462-1469.

21. Marocolo IC, da Mota GR, Londe AM, Patterson SD, Barbosa Neto O, Marocolo M. Acute ischemic preconditioning does not influence highintensity intermittent exercise performance. Peer J. 2017:5:e4118.

22. Kjeld T, Rasmussen MR, Jattu T, Nielsen HB, Secher NH. Ischemic preconditioning of one forearm enhances static and dynamic apnea. Medicine and science in sports and exercise. Jan 2014;46(1):151-155

23. Beaven CM, Cook CJ, Kilduff L, Drawer S, Gill N. Intermittent lower-limb occlusion enhances recovery after strenuous exercise. Applied physiology, nutrition, and metabolism = Physiologie appliquee, nutrition et metabolisme. Dec 2012;37(6):1132-1139.

24. Cochrane DJ, Booker HR, Mundel T, Barnes MJ. Does intermittent pneumatic leg compression enhance muscle recovery after strenuous eccentric exercise? International journal of sports medicine. Nov 2013;34(11):969-974

25. Marocolo M, Coriolano HA, Mourao CA da Mota GR. Crucial Points for Analysis of Ischemic Preconditioning in Sports and Exercise. Medicine and science in sports and exercise. Jul 2017;49(7):1495-1496.

26. Krustrup $P$, Mohr M, Amstrup T, et al. The yo-yo intermittent recovery test: physiological response, reliability, and validity. Medicine and science in sports and exercise. Apr 2003;35(4):697-705.

27. Simim MAM, Silva BVC, Marocolo Júnior M, Mendes EL, Mello MTd, Mota GRd. Anthropometric profile and physical performance characteristic of the Brazilian amputee football (soccer) team. Motriz: Revista de Educação Física. 2013;19:641-648

28. Andersson $\mathrm{H}$, Ekblom B, Krustrup P. Elite football on artificial turf versus natural grass: movement patterns, technical standards, and player impressions. Journal of sports sciences. Jan 15 2008;26(2):113-122.

29. Smith MR, Zeuwts L, Lenoir M, Hens N, De Jong LM, Coutts AJ. Mental fatigue impairs soccer-specific decision-making skill. Journal of sports sciences. Jul 2016;34(14):1297-1304.

30. da Mota GR, Thiengo CR, Gimenes SV, Bradley PS. The effects of ball possession status on physical and technical indicators during the 2014 FIFA World Cup Finals. Journal of sports sciences. 2016;34(6):493-500.

31. Scarfone R, Ammendolia A. Match analysis of an elite beach soccer team. The Journal of sports medicine and physical fitness. Jul-Aug 2017;57(7 8):953-959.

32. Simim MAM, da Mota GR, Marocolo M, da Silva BVC, de Mello MT, Bradley PS. The Demands of Amputee Soccer Impair Muscular Endurance and Power Indices But Not Match Physical Performance. Adapted physical activity quarterly: APAQ. Jan 5 2018:1-17.

33. Simim MA, Bradley PS, da Silva BV, et al. The quantification of gameinduced muscle fatigue in amputee soccer players. The Journal of sports medicine and physical fitness. Jun 2017;57(6):766-772.

34. Wilke CF, Ramos GP, Pacheco DA, et al. Metabolic Demand and Interna Training Load in Technical-Tactical Training Sessions of Professional Futsal Players. Journal of strength and conditioning research. Aug 2016:30(8):2330-2340

35. Simim MAM, da Silva BVC, da Mota GR. Futebol para amputados: aspectos técnicos, táticos e diretrizes para o treinamento. Revista Brasileira de Futsal e Futebol RBFF 2015;7(25):246-254

36. Marocolo M, Marocolo IC, da Mota GR, Simao R, Maior AS, Coriolano HJ Beneficial Effects of Ischemic Preconditioning in Resistance Exercise Fade Over Time. International journal of sports medicine. Sep 2016;37(10):819824

37. Beedie CJ, Foad AJ. The placebo effect in sports performance: a brief review. Sports Med. 2009;39(4):313-329.

38. da Silva BV, Ide BN, de Moura Simim MA, Marocolo M, da Mota GR Neuromuscular responses to simulated brazilian jiu-jitsu fights. Journal of human kinetics. Dec 9 2014:44:249-257.

39. da Silva BV, Simim MA, Marocolo M, Franchini E, da Mota GR. Optimal load for the peak power and maximal strength of the upper body in Brazilian Jiu-Jitsu athletes. Journal of strength and conditioning research. Jun 2015;29(6):1616-1621.

40. da Mota GR, Magalhães CG, Azevedo PHSM, et al. Lactate threshold in taekwondo through specifics tests. Journal of Exercise Physiology online. 2011;14(3):60-66

41. Meneghel AJ, Verlengia R, Crisp AH, et al. Muscle damage of resistancetrained men after two bouts of eccentric bench press exercise. Journal of strength and conditioning research. Oct 2014;28(10):2961-2966.

42. Maior AS, Paixão RC, Ribeiro IC, Freitas DGS, da Mota GR, Marocolo M Acute responses of rate pressure product in sets of resistance exercise. Medicina Sportiva. 2014;18:36-41

43. Calixto $R$, Verlengia R, Crisp A, et al. Acute effects of movement velocity on blood lactate and growth hormone responses after eccentric bench press exercise in resistance-trained men. Biology of sport. Dec 2014;31(4):289-294

44. Ide BN, Leme TC, Lopes CR, et al. Time course of strength and power recovery after resistance training with different movement velocities. Journal of strength and conditioning research. Jul 2011;25(7):2025-2033.

45. da Mota GR, Barbosa Neto O, Faleiros ACG, et al. Street-dance: Physiological demands and effect of endurance training. Journal of Physical Education and Sports Management. 2011;2(5):53-57. 